OPEN ACCESS

Edited by:

Błażej Misiak,

Wroclaw Medical University, Poland

Reviewed by:

Elena Martín-García,

Universidad Pompeu Fabra, Spain

Milica Milovan Borovcanin,

University of Kragujevac, Serbia

*Correspondence:

Tohru Ohnuma

otoru@juntendo.ac.jp

Specialty section:

This article was submitted to

Psychopharmacology,

a section of the journal

Frontiers in Psychiatry

Received: 21 November 2017

Accepted: 28 February 2018

Published: 13 March 2018

Citation:

Ohnuma T, Nishimon S, Takeda M,

Sannohe T, Katsuta N and Arai $H$ (2018) Carbonyl Stress and Microinflammation-Related Molecules as Potential Biomarkers in Schizophrenia.

Front. Psychiatry 9:82 doi: 10.3389/fpsyt.2018.00082

\section{Carbonyl Stress and} Microinflammation-Related Molecules as Potential Biomarkers in Schizophrenia

\author{
Tohru Ohnuma*, Shohei Nishimon, Mayu Takeda, Takahiro Sannohe, Narimasa Katsuta \\ and Heii Arai \\ Juntendo University Schizophrenia Projects (JUSP), Department of Psychiatry, Faculty of Medicine, Juntendo University, \\ Tokyo, Japan
}

This literature review primarily aims to summarize our research, comprising both cross-sectional and longitudinal studies, and discuss the possibility of using microinflammation-related biomarkers as peripheral biomarkers in the diagnosis and monitoring of patients with schizophrenia. To date, several studies have been conducted on peripheral biomarkers to recognize the potential markers for the diagnosis of schizophrenia and to determine the state and effects of therapy in patients with schizophrenia. Research has established a correlation between carbonyl stress, an environmental factor, and the pathophysiology of neuropsychiatric diseases, including schizophrenia. In addition, studies on biomarkers related to these stresses have achieved results that are either replicable or exhibit consistent increases or decreases in patients with schizophrenia. For instance, pentosidine, an advanced glycation end product (AGE), is considerably elevated in patients with schizophrenia; however, low levels of vitamin B6 [a detoxifier of reactive carbonyl compounds (RCOs)] have also been reported in some patients with schizophrenia. Another study on peripheral markers of carbonyl stress in patients with schizophrenia revealed a correlation of higher levels of glyceraldehyde-derived AGEs with higher neurotoxicity and lower levels of soluble receptors capable of diminishing the effects of AGEs. Furthermore, studies on evoked microinflammation-related biomarkers (e.g., soluble tumor necrosis factor receptor 1) have reported relatively consistent results, suggesting the involvement of microinflammation in the pathophysiology of schizophrenia. We believe that our cross-sectional and longitudinal studies as well as various previous inflammation marker studies that could be interpreted from several perspectives, such as mild localized encephalitis and microvascular disturbance, highlighted the importance of early intervention as prevention and distinguished the possible exclusion of inflammations in schizophrenia.

Keywords: AGEs, biomarkers, carbonyl stress, glyceraldehyde-derived AGEs, microinflammation, pentosidine, schizophrenia, soluble tumor necrosis factor receptor 1

\section{INTRODUCTION}

Several studies have been performed to identify peripheral biomarkers for use in the diagnosis and monitoring of schizophrenia. These have mostly been based on pathophysiological hypotheses that schizophrenia is caused by disturbed neurotransmission, such as the dopaminergic (1) and glutamatergic (2-5) hypothesis, and thus have investigated the potential roles of peripheral 
monoamines and amino acids. Other studies have also investigated molecules related to the neurodevelopmental hypothesis, such as brain-derived neurotrophic factors $(6,7)$. Although some of these studies have shown altered biomarker levels in patients with schizophrenia, consistent results have not been achieved on replication, thereby raising questions over the validity of their use as diagnostic or therapeutic biomarkers (8-10). In addition, these studies have failed to show whether endogenous monoamine and/or amino acid levels in the peripheral blood truly reflect brain levels.

An alternative approach, based on the role of environmental factors, has also been proposed. Indeed, oxidative stress (11-13) and carbonyl stress (14-17), both environmental factors, have been associated with the pathophysiology of schizophrenia. Studies of biomarkers related to these stresses have achieved either results that are replicable or at least in the same direction (increases or decreases), concerning altered biomarker levels in schizophrenia. Moreover, relatively consistent results have been demonstrated in evoked microinflammation-related biomarker studies, with a subset of patients with schizophrenia showing pathophysiological microinflammation. In the present literature review, we discuss the findings of our previous studies, comprised of not only cross-sectional research, but also large-scale, longitudinal observations in which we identified putative biomarkers in the peripheral blood. We propose that these could be used for the diagnosis and monitoring of subpopulations of patients with schizophrenia.

\section{CARBONYL STRESS}

\section{Pentosidine and Pyridoxal}

Interesting results from a cross-sectional study showed that plasma levels of pentosidine, an advanced glycation end product (AGE), were significantly increased in patients with schizophrenia and that a subpopulation had low levels of vitamin B6 (14). In carbonyl stress pathway, reactive carbonyl compounds (RCOs), which cause carbonyl stress, are detoxified by degradation into lactic acid and glutathione by glyoxalase enzymes. Glyoxalase 1 and 2 (GLO1 and GLO2) are the rate-limiting enzymes in this metabolic pathway. Inhibition of RCO generation and the Maillard reaction by vitamin B6 results in the suppression of AGE accumulation (Figure 1A). This is important because vitamin B6 detoxifies RCOs. Among those with high pentosidine levels, most were also shown to have a family history of psychiatric illness, severe symptoms, and an affected gene associated with RCOs (14). A subsequent cross-sectional study that included more clinical data and more patients with chronic schizophrenia showed that the presence of carbonyl stress could lead to treatment resistance, establishing a role for markers carbonyl stress in chronic schizophrenia (16). These studies evidenced that high pentosidine and low pyridoxal levels in the peripheral blood could be state markers of "treatment resistance" in some patients with schizophrenia. However, to verify whether altered pentosidine and pyridoxal levels could be "state" and/or "therapeutic" biological markers of schizophrenia, parallel cross-sectional and longitudinal studies were needed that followed patients with schizophrenia from acute illness to remission.

\section{Pentosidine}

We repeated our original cross-sectional study to investigate the means and clinical significance of serum markers of carbonyl stress in 137 patients with acute schizophrenia and in 47 healthy controls (15). Although serum pentosidine levels were markedly elevated in some patients, levels were not significantly altered in schizophrenia (Figure 1B), meaning that pentosidine could not be confirmed as a state biomarker for severity. Moreover, the putative marker showed no correlation with any other clinical feature of schizophrenia (e.g., age at onset, illness duration, and family history). There was, however, a significant positive correlation between pentosidine levels and both the daily antipsychotic dose and the cumulative antipsychotic exposure (duration multiplied by the daily dose) (15).

The positive correlation between pentosidine levels and daily antipsychotic dose has been reported in previous studies (14-17), which concluded that this was due to the characteristic that treatment-resistant patients are usually treated with relatively high daily antipsychotic doses. To test this hypothesis, the influence of first- and second-generation antipsychotics, as well as the influence of different combinations of medication and dosages, was analyzed in a follow up to our first cross-sectional study (15). We enrolled another 137 patients with acute schizophrenia and pooled them with the existing cohort (22). We evaluated the associations of serum pentosidine with clinical variables that have shown a significant association with peripheral pentosidine levels (14-17). These included the severity of symptoms, the duration of education, and the duration and daily doses of antipsychotic, antiparkinsonian, and anxiolytic medications. The pooled cohort $(n=274)$ showed associations between higher serum pentosidine levels and both higher daily antipsychotic doses and longer estimated durations of medication use, only in the context of antipsychotic polypharmacy (not with monotherapy). However, there was no statistical significance for diagnostic purposes (Table 1) (22).

\section{Pyridoxal}

As mentioned, our first cross-sectional study also showed a potential role for low levels of pyridoxal, a form of vitamin B6 (15). In the first replication study (Figure 1C) (15) with a pooled cohort of 274 patients (22), the results were consistent, showing a significant decrease in pyridoxal levels among patients with schizophrenia. Although no study had shown a correlation with other clinical features of the disease $(14-17,22)$ in our longitudinal study, we managed to show that the low pyridoxal levels during acute schizophrenia increased according to the clinical course of the illness but were not directly correlated with symptom improvement. Moreover, 18 patients whose pyridoxal levels decreased during their illnesses had less symptom improvement (Figure 1D) (15). Thus, we concluded that decreasing pyridoxal levels during the clinical course of schizophrenia could be a biomarker for non-responders to antipsychotic therapy.

\section{Glyceraldehyde-Derived AGEs (Glycer- AGEs)}

Recently, strong in vivo neurotoxicity has been shown with Glycer-AGEs (23) that are central to the pathophysiology of 


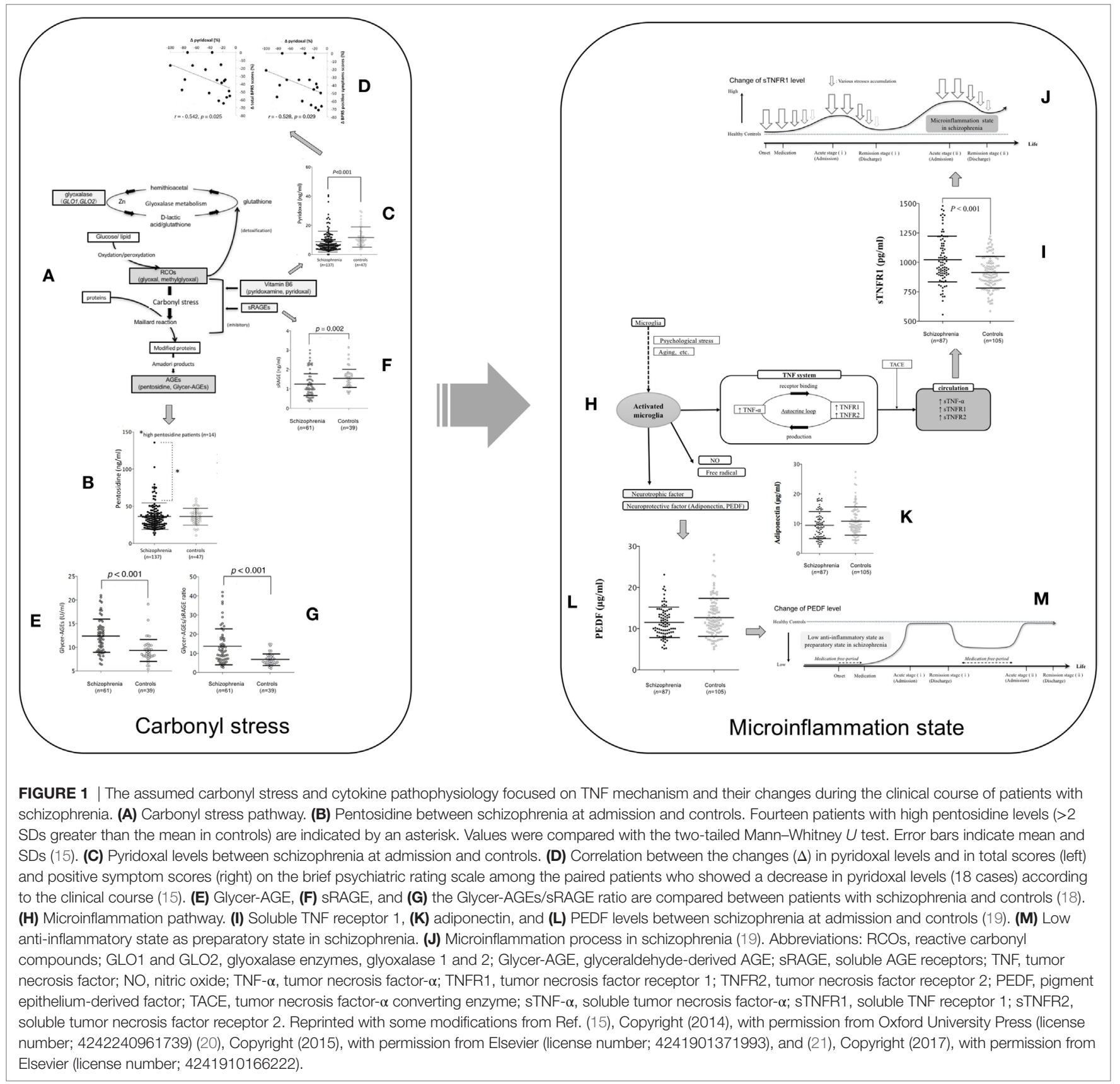

neurodegenerative diseases $(18,24)$. In a longitudinal crosssectional study of peripheral serum Glycer-AGE levels, we included 61 patients with acute schizophrenia and 39 controls, with follow up data for 54 patients to remission (20). Peripheral Glycer-AGE levels were significantly higher than those of controls (Figure 1E), but did not change with the clinical disease course and were not correlated with clinical features, indicating that they may not be useful as therapeutic or state markers in patients with schizophrenia (20); however, they could serve as diagnostic markers. Indeed, unlike pentosidine, their levels were not correlated with daily chlorpromazine doses, indicating the lack of an iatrogenic effect (15).

\section{Soluble Receptors for AGE Receptors}

AGEs interact with AGE receptors (RAGEs) to increase oxidative and carbonyl stress (25). Circulating receptors are also bound, such as endogenous secretory RAGE (esRAGE) and soluble receptors for RAGE (sRAGE), with the latter most likely to be indicative of carbonyl stress because they exist at levels five times greater than those of esRAGE (25). Peripheral serum soluble AGE receptors (sRAGE) levels were investigated in the earlier study of Glycer-AGEs (20), where levels in patients with acute schizophrenia were found to be significantly lower than those in healthy controls (Figure 1F). Accordingly, significant negative correlations were identified between serum Glycer-AGE and sRAGE 
TABLE 1 | Multiple linear regression analysis of possible explanatory variables for the serum pentosidine levels.

\begin{tabular}{|c|c|c|c|c|}
\hline \multirow[b]{3}{*}{ Independent variables: } & \multirow{2}{*}{\multicolumn{2}{|c|}{$\begin{array}{c}\text { Total } \\
(N=274)\end{array}$}} & \multirow{2}{*}{\multicolumn{2}{|c|}{$\begin{array}{c}\text { Poly } \\
(N=68)\end{array}$}} \\
\hline & & & & \\
\hline & $b(\mathrm{SE})$ & $\boldsymbol{\beta}$ & $b(\mathrm{SE})$ & $\boldsymbol{\beta}$ \\
\hline Duration of education & - & - & - & - \\
\hline Total BPRS score & - & - & - & - \\
\hline $\begin{array}{l}\text { Estimated duration } \\
\text { of medication }\end{array}$ & $0.318(0.139)$ & $0.197^{*}$ & $0.900(0.443)$ & $0.365^{\star}$ \\
\hline Daily dose of antipsychotics & $0.012(0.003)$ & $0.390^{\star \star \star}$ & $0.014(0.007)$ & $0.368^{*}$ \\
\hline $\begin{array}{l}\text { Daily dose of } \\
\text { antiparkinsonian drugs }\end{array}$ & - & - & - & - \\
\hline Daily dose of anxiolytics & - & - & - & - \\
\hline $\begin{array}{l}\text { Constant } \\
R^{2}\end{array}$ & $\begin{array}{l}32.0 \\
0.195\end{array}$ & & $\begin{array}{l}16.2 \\
0.407^{\star \star \star}\end{array}$ & \\
\hline
\end{tabular}

Multiple linear regression analysis includes six possible explanatory variables for all 274 patients and for the polypharmacy treatment group. Statistical values of independent factors excluded from the first step of the equation are not shown. ${ }^{*} p<0.05$,

${ }^{* *} p<0.01,{ }^{* *} p<0.001(22)$.

BPRS, brief psychiatric rating scale; Poly, polypharmacy treatment group (treated with both types of antipsychotics; at least one of each); SE; standard error. Reprinted from Ref. (20), Copyright (2017), with permission from Elsevier (license number; 4241900807314).

levels, with Glycer-AGEs/sRAGE ratios being sensitive markers of carbonyl stress. Indeed, the ratio differed significantly, with an approximately twofold higher ratio in patients with schizophrenia than in healthy controls (Figure 1G). Neither sRAGE levels nor Glycer-AGEs/sRAGE ratios showed any correlation with clinical symptoms or change with the clinical course, meaning that they could not be used as state makers of schizophrenia.

Interestingly, discriminant analyses confirmed that GlycerAGEs and Glycer-AGEs/sRAGE ratios were significant diagnostic markers for schizophrenia, effectively distinguishing between patients and controls in 70\% of cases. Thus, Glycer-AGEs and their ratio to sRAGE could be used as diagnostic markers of schizophrenia.

\section{MICROINFLAMMATION}

The oxidative $(11,26)$ and carbonyl stresses $(15,20,22)$ involved in the pathophysiology of schizophrenia have been considered to induce a proinflammatory state that could lead to microinflammation (Figure 1H). In microinflammation pathway, when the brain is exposed to psychological stress or aging, the microglia develop to an activated state. The activated microglia release various inflammatory cytokines, nitric oxide, free radicals, neurotrophic factor, and neuroprotective factor. Tumor necrosis factor-alpha (TNF- $\alpha$ ) binds to TNFR1 and TNFR2 and the TNFRs induce TNF- $\alpha$ production. These "autocrine loops" chronically continue to act on the TNF system. TNF- $\alpha$, TNFR1, and TNFR2 are cleaved by TNF- $\alpha$ converting enzyme, and subsequently exist as soluble tumor necrosis factor- $\alpha$, soluble TNF receptor 1 (sTNFR1), and sTNF2 in circulation. These systems ultimately induce neuroinflammation. Several investigations of inflammatory markers in the peripheral blood have assessed the chronic inflammatory statuses of patients with schizophrenia (27-30).
In a recent review and meta-analysis, interleukin (IL)-1 $\beta$, IL-6, and transforming growth factor- $\beta$ were identified as putative state markers, whereas IL-12, interferon- $\gamma$, tumor necrosis factor (TNF)- $\alpha$, and soluble IL-2 receptors were identified as putative trait markers based on evidence from longitudinal observations of patients with acute schizophrenia (31). However, whether changes in these biomarker levels reflect the current pathological disease state has not been established when controlled for age, sex, and body mass index (BMI). It is, therefore, unclear if these can be useful as biological markers for acute schizophrenia in clinical practice. In our recent longitudinal cross-sectional study of microinflammatory biomarkers, we investigated whether serum levels of sTNFR1, adiponectin, and pigment epithelium-derived factor (PEDF) could be used as diagnostic and/or prognostic biomarkers for acute schizophrenia.

\section{Soluble TNF Receptor 1}

Serum TNF- $\alpha$ has been shown to have proinflammatory effects. These effects occur when it binds to TNF receptors 1 or 2 (32), which enter the circulation as soluble TNFRs (sTNFRs) (33). Given that the most stable and reliable marker of TNF- $\alpha$ activity is considered to be sTNFR1 $(34,35)$, biomarker studies were done to investigate the average levels (means) of neuroinflammation in patients with schizophrenia $(19,34-36)$, concluding that sTNFR1 could reflect treatment resistance or severe clinical disease trajectories $(19,35)$.

In cross-sectional research, we reported significant differences in peripheral sTNFR1 levels between patients with acute schizophrenia and controls, regardless of whether we adjusted for physical confounders that could affect microinflammation (e.g., BMI and age) (21). Peripheral sTNFR1 levels were significantly higher in the patients with acute schizophrenia than in healthy controls (Figure 1I), but high sTNFR1 alone showed moderate discriminating efficacy between the two groups ( $>60 \%$ accuracy). The marker also effectively discriminated healthy controls from patients with clinical deterioration ( $>90 \%$ accuracy) and from patients with clinical improvement ( $>80 \%$ accuracy) during inpatient care. Thus, higher serum sTNFR1 levels could predict so-called treatment-resistant schizophrenia, as supported by the results of previous studies $(19,35)$. Although the peripheral sTNFR1 levels did not reflect clinical severity in acute schizophrenia, levels were correlated with the duration of illness and age, consistent with previous research showing an association between inflammatory biomarkers and age in patients with psychiatric disease (27). However, healthy controls showed no such association between age and peripheral sTNFR1 levels, and patients who were not taking medication had significantly shorter illness durations and lower sTNFR1 levels compared with medicated patients (21). Thus, we concluded that sTNFR1 levels may only increase as the disease progresses and stressors accumulate (Figure 1J) (21).

In longitudinal research, we found that intensive antipsychotic therapy between admission and discharge produced significant decreases in elevated peripheral sTNFR1 levels (21). Unfortunately, sTNFR1 did not reflect symptomatic improvement, with changes in levels failing to correlate with changes in positive, negative, or total scores on the Brief Psychiatric Rating 
Scale, scores on the Global Assessment of Functioning scale, or with daily antipsychotic doses. We, therefore, concluded that overall relief of psychological stress factors was reflected by changes in peripheral sTNFR1 levels, with abating symptoms being only one such factor. Importantly, hospitalization did not appear to be a cause of stress (Figure 1J).

\section{Adiponectin}

Adiponectin has been shown to regulate insulin sensitivity and tissue inflammation (37), but in our studies, we found no significant differences in adiponectin levels, irrespective of whether it was matched for potential confounders (Figure 1K). However, there was a significant correlation between BMI and serum adiponectin levels independent of the disease (21). Several studies have indicated that peripheral adiponectin levels are elevated in patients with chronic schizophrenia, concluding that adiponectin could be a marker for the metabolic syndrome for schizophrenia, especially that triggered by atypical antipsychotics, rather than reflecting the disease (38-42).

\section{Pigment Epithelium-Derived Factor}

Pigment epithelium-derived factor inhibits the AGE-RAGE pathway in the activation of proinflammatory genes, with higher peripheral AGE/sRAGE ratios reported in patients with schizophrenia (20). By suppressing proinflammatory pathways, such as those involved in carbonyl stress, PEDF may be able to inhibit inflammation (43), and may function as an antiinflammatory in acute schizophrenia. In the above-mentioned study of sTNFR1 (21), peripheral PEDF levels were not significantly different between patients with acute schizophrenia and controls, regardless of matching for confounders (e.g., BMI and age; Figure 1L). Interestingly, peripheral PEDF levels in the 42 patients not receiving medication were significantly lower compared with controls, but without producing higher sTNFR1 levels (21). It is possible that the lower PEDF levels in untreated patients with shorter disease durations reflect a preparatory state for an inflammatory pathophysiology, from which prolonged and severe disease-related stress causes chronic inflammation reflected by increased peripheral sTNFR1 in patients receiving antipsychotics (Figure 1M). In addition, the fact that PEDF did not alter over time and did not correlate with other clinical variables may indicate that lower levels only affect disease onset (Figure 1M) (21). Taken together mentioned-above cross-sectional and longitudinal changes in sTNFR1 and PEDF levels in schizophrenia, we hypothesized that; while all patients with schizophrenia were initially treated with medication on admission, it was noted that patients not taking medications, including drug-naïve patients and those with relapse, had low PEDF levels (Figure 1M), but similar sTNFR1 levels (Figure 1J) when compared with healthy controls. This indicated a low anti-inflammatory state among these patients. However, during the acute exacerbation, greater stress levels (Figure 1J; arrows) were associated with higher sTNFR1 levels compared to healthy controls. Subsequently, while sTNFR1 and psychological stress levels decreased by the time of remission, PEDF levels did not change despite intensive antipsychotic therapy (Figure 1M).

\section{Involvement of Carbonyl Stress and Microinflammation in Schizophrenia: Model of Mild Localized Encephalitis With Microvascular Damage}

Several epidemiological studies have suggested the involvement of prenatal (in utero) infection-related inflammation in the pathophysiology of schizophrenia $(44,45)$. In particular, a study highlighted the prenatal exposure to herpes simplex virus type 1 infection as one of the etiologies of onset (46). Clinically, patients with mild localized encephalitis, especially localized temporal lobe encephalitis caused by herpes simplex virus type 1 , have been shown to exhibit schizophrenic-like symptoms, such as auditory hallucinations and cognitive impairments (47-49). Environmental factors, such as physical stress (including these infections), hypoxia, and oxidative as well as carbonyl stress are known to evoke microinflammation that can damage the neurons and microvascular systems, with the latter damage easily (50-52). Reportedly, these findings could corroborate the already hypothesized "vascular-inflammatory theory" in the central nervous system of patients with schizophrenia (53). Despite the relatively mild degree of inflammation caused by each environmental factor, the cumulative microinflammation due to the repeated exposure to various postnatal stresses could evoke the exacerbation and/or treatment resistance in patients with schizophrenia (Figure 1J). Overall, while the damage to temporal lobe microvascular system might be only partly involved in the pathophysiology of onset of schizophrenia, the prevention of exposure to these microinflammations could be essential in patients with schizophrenia.

\section{Microinflammations as Specific Pathophysiology in Schizophrenia}

Reportedly, environmental stress factors and evoked microinflammations could be involved in several psychiatric disorders, such as depression (54) and bipolar disorders, and some of these disorders exhibit characteristics similar to those exhibited in schizophrenia with altered cytokines (36). For instance, not only apparent neurodegenerative diseases, but also other psychiatric disorders could involve altered cytokine systems as their pathophysiology. In fact, patients with post-traumatic stress disorder, typically caused only due to intense psychological stress, also exhibited an elevation in peripheral TNF- $\alpha$ levels, which were reproducibly reported in other psychiatric diseases as well, including schizophrenia $(36,55)$. Thus, further studies are warranted to investigate the difference in the degree of alteration of cytokine levels among various neuropsychiatric disorders. Perhaps, findings of future research could be a diagnostic marker in the clinical practice if the discriminant analysis established a significant difference between several subjects and same measurement methods.

\section{Immune Responses Affected by Microinflammation in Schizophrenia}

Although the immune responses directly affected by aforementioned stresses cannot be inferred, an interesting epidemiological study established an association of atopic disorders (in general) 
and asthma (in particular), namely type 1 hypersensitivity, with the risk of developing schizophrenia (56). In addition, elevated type-2 cytokine levels in schizophrenia were also reported to be a part of the pathophysiology (57). While psychosocial stress alone also could lead to asthma exacerbation by accompanying with histological microvascular system inflammation and increase of serum type- 2 cytokine levels in asthma model animal (58). Taken together these findings, the damaged microvascular system seems to be the crucial site of inflammation in asthma and in the schizophrenia, especially in exacerbation.

Based on our previous studies and other above-mentioned studies on psychiatric subjects, increased TNF superfamily levels, among several cytokines, could be, at least, involved in the pathophysiology of psychiatric diseases (Figure 1H).

\section{Early Prediction and Prevention of Microinflammation}

Among markers of microinflammation, the most reproducible outcomes have been obtained for elevated serum sTNFR1 levels as a marker of treatment resistance in patients with schizophrenia $(21,34,36)$. Apparently, early intervention for diseases associated with carbonyl stress and microinflammation, such as diabetes mellitus, is imperative to prevent irreversible complications. Consequently, earlier discovery of proinflammatory states with sensitive markers of carbonyl stress, before any disease reaches the inflammatory stage, could be clinically beneficial. Reportedly, regarding the carbonyl stress status, Glycer-AGEs exert strong neurotoxicity (23), and Glycer-AGEs as well as the Glycer-AGE:sRAGE ratio demonstrate higher sensitivities than other AGEs-related molecules in schizophrenia (20). Thus, serum Glycer-AGEs and the GlycerAGE:sRAGE ratio could act as important proinflammatory markers of subsequent microinflammation that is potentially associated with treatment-resistant schizophrenia. In addition, some studies have revealed that early intervention for prevention of exposure to these stresses, e.g., diet and habits, as well as a higher exposure to the daily dose of antipsychotics $(22,59)$ would be beneficial for such patients. Recently, an interesting study reported that augmentation therapy with high-dose pyridoxamine (a form of vitamin B6 that detoxifies RCOs) could improve, in part, patients with high AGEs levels and be a novel strategy for treatment-resistant schizophrenia (60). Furthermore, a study reporting that for patients in a state of micronflammation, add-on-therapy with Cox-II-blockers (nonsteroidal anti-inflammatory drugs) or valacyclovir (an antiviral drug), which improved acute schizophrenia, could be used as a treatment strategy based on the above-mentioned mild localized encephalitis model (61).

\section{REFERENCES}

1. Davis KL, Kahn RS, Ko G, Davidson M. Dopamine in schizophrenia: a review and reconceptualization. Am J Psychiatry (1991) 148:1474-86. doi:10.1176/ ajp.148.11.1474

2. Tortorella A, Monteleone P, Fabrazzo M, Viggiano A, De Luca L, Maj M. Plasma concentrations of amino acids in chronic schizophrenics treated

\section{CONCLUSION}

In this review, we primarily offered a summary and analysis of our previous research into markers of proinflammatory carbonyl stress and microinflammation, and now highlight six key conclusions. First, in patients receiving antipsychotic polypharmacy, high peripheral pentosidine levels may be associated with high daily doses and longer antipsychotic use. Second, decreasing pyridoxal levels during treatment could potentially identify nonresponders to antipsychotic therapy. Third, although apparently unsuitable for use as markers of proinflammatory states, higher Glycer-AGE levels and higher Glycer-AGEs/sRAGE ratios could be diagnostic of schizophrenia. Fourth, as previously reported $(19,35)$, elevated sTNFR1 levels are accurate at discriminating patients who deteriorate during inpatient care from both healthy controls and patients who improve. Thus, higher peripheral sTNFR1 levels may not only be a useful adjunctive diagnostic biomarker for acute schizophrenia, but may also be a valuable prognostic biomarker for treatment response. Fifth, low PEDF levels in untreated patients with short disease durations might reflect a preparatory state for inflammation. Finally, raised adiponectin levels could be useful as a marker of the metabolic syndrome in patients receiving antipsychotics for schizophrenia. Our cross-sectional and longitudinal studies as well as various previous inflammation marker studies that could be interpreted from several perspectives, such as mild localized encephalitis and microvascular disturbance, highlighted the importance of early intervention as prevention and distinguished the possible exclusion of inflammations in schizophrenia.

\section{AUTHOR CONTRIBUTIONS}

TO contributed to the interpretation of the data and writing of the paper. SN, MT, TS, and NK contributed to the clinical evaluation of patients and the conception of the study. HA contributed to the conception and design of the study. All authors contributed to and approved the final manuscript.

\section{ACKNOWLEDGMENTS}

The authors would like to thank Enago (www.enago.jp) for their English language review. The technical assistance of Mrs. K. Yamamoto and Ms. W. Mao for measurement of carbonyl stress markers is also gratefully acknowledged.

\section{FUNDING}

This work was supported by the Juntendo Institute of Mental Health from 2016 to 2017 (201607).

with clozapine. Neuropsychobiology (2001) 44:167-71. doi:10.1159/ 000054937

3. Maeshima H, Ohnuma T, Sakai Y, Shibata N, Baba H, Ihara H, et al. Increased plasma glutamate by antipsychotic medication and its relationship to glutaminase 1 and 2 genotypes in schizophrenia - Juntendo University Schizophrenia Projects (JUSP). Prog Neuropsychopharmacol Biol Psychiatry (2007) 31:1410-8. doi:10.1016/j.pnpbp.2007.06.009 
4. Palomino A, Gonzalez-Pinto A, Aldama A, Gonzalez-Gomez C, Mosquera F, Gonzalez-Garcia G, et al. Decreased levels of plasma glutamate in patients with first-episode schizophrenia and bipolar disorder. Schizophr Res (2007) 95:174-8. doi:10.1016/j.schres.2007.06.012

5. Ohnuma T, Shibata N, Maeshima H, Baba H, Hatano T, Hanzawa R, et al. Association analysis of glycine- and serine-related genes in a Japanese population of patients with schizophrenia. Prog Neuropsychopharmacol Biol Psychiatry (2009) 33:511-8. doi:10.1016/j.pnpbp.2009.02.004

6. Zhang XY, Liang J, Chen DC, Xiu MH, De Yang F, Kosten TA, et al. Low BDNF is associated with cognitive impairment in chronic patients with schizophrenia. Psychopharmacology (Berl) (2012) 222(2):277-84. doi:10.1007/ s00213-012-2643-y

7. Zugman A, Pedrini M, Gadelha A, Kempton MJ, Noto CS, Mansur RB, et al. Serum brain-derived neurotrophic factor and cortical thickness are differently related in patients with schizophrenia and controls. Psychiatry Res (2015) 234:84-9. doi:10.1016/j.pscychresns.2015.08.009

8. Weickert CS, Weickert TW, Pillai A, Buckley PF. Biomarkers in schizophrenia: a brief conceptual consideration. Dis Markers (2013) 35:3-9. doi:10.1155/2013/510402

9. Green MJ, Matheson SL, Shepherd A, Weickert CS, Carr VJ. Brain-derived neurotrophic factor levels in schizophrenia: a systematic review with meta-analysis. Mol Psychiatry (2011) 16:960-72. doi:10.1038/mp.2010.88

10. Ohnuma T, Arai H. Significance of NMDA receptor-related glutamatergic amino acid levels in peripheral blood of patients with schizophrenia. Prog Neuropsychopharmacol Biol Psychiatry (2011) 35:29-39. doi:10.1016/j. pnpbp.2010.08.027

11. Marchbanks RM, Ryan M, Day IN, Owen M, McGuffin P, Whatley SA. A mitochondrial DNA sequence variant associated with schizophrenia and oxidative stress. Schizophr Res (2003) 65:33-8. doi:10.1016/S0920-9964(03)00011-2

12. Prabakaran S, Swatton JE, Ryan MM, Huffaker SJ, Huang JT, Griffin JL, et al. Mitochondrial dysfunction in schizophrenia: evidence for compromised brain metabolism and oxidative stress. Mol Psychiatry (2004) 9(7):684-97. doi:10.1038/sj.mp.4001532

13. Yao JK, Reddy RD, van Kammen DP. Oxidative damage and schizophrenia: an overview of the evidence and its therapeutic implications. CNS Drugs (2001) 15:287-310. doi:10.2165/00023210-200115040-00004

14. Arai M, Yuzawa H, Nohara I, Ohnishi T, Obata N, Iwayama Y, et al. Enhanced carbonyl stress in a subpopulation of schizophrenia. Arch Gen Psychiatry (2010) 67:589-97. doi:10.1001/archgenpsychiatry.2010.62

15. Katsuta N, Ohnuma T, Maeshima H, Takebayashi Y, Higa M, Takeda M, et al. Significance of measurements of peripheral carbonyl stress markers in a cross-sectional and longitudinal study in patients with acute-stage schizophrenia. Schizophr Bull (2014) 40:1366-73. doi:10.1093/schbul/sbt234

16. Miyashita M, Arai M, Kobori A, Ichikawa T, Toriumi K, Niizato K, et al. Clinical features of schizophrenia with enhanced carbonyl stress. Schizophr Bull (2014) 40:1040-6. doi:10.1093/schbul/sbt129

17. Miyashita M, Arai M, Yuzawa H, Niizato K, Oshima K, Kushima I. Replication of enhanced carbonyl stress in a subpopulation of schizophrenia. Psychiatry Clin Neurosci (2014) 68:83-4. doi:10.1111/pcn.12081

18. Takeuchi M, Yamagishi S. Involvement of toxic AGEs (TAGE) in the pathogenesis of diabetic vascular complications and Alzheimer's disease. J Alzheimers Dis (2009) 16:845-58. doi:10.3233/JAD-2009-0974

19. Noto C, Maes M, Ota VK, Teixeira AL, Bressan RA, Gadelha A, et al. High predictive value of immune-inflammatory biomarkers for schizophrenia diagnosis and association with treatment resistance. World J Biol Psychiatry (2015) 27:1-8. doi:10.3109/15622975.2015.1062552

20. Takeda M, Ohnuma T, Takeuchi M, Katsuta N, Maeshima H, Takebayashi Y, et al. Altered serum glyceraldehyde-derived advanced glycation end product (AGE) and soluble AGE receptor levels indicate carbonyl stress in patients with schizophrenia. Neurosci Lett (2015) 593:51-5. doi:10.1016/j. neulet.2015.03.002

21. Nishimon S, Ohnuma T, Takebayashi Y, Katsuta N, Takeda M, Nakamura T, et al. High serum soluble tumor necrosis factor receptor 1 predicts poor treatment response in acute-stage schizophrenia. Prog Neuropsychopharmacol Biol Psychiatry (2017) 76:145-54. doi:10.1016/j.pnpbp.2017.03.006

22. Sannohe T, Ohnuma T, Takeuchi M, Tani E, Miki Y, Takeda M, et al. High doses of antipsychotic polypharmacy are related to an increase in serum levels of pentosidine in patients with schizophrenia. Prog Neuropsychopharmacol Biol Psychiatry (2017) 76:42-8. doi:10.1016/j.pnpbp.2017.02.019
23. Takeuchi M, Bucala R, Suzuki T, Ohkubo T, Yamazaki M, Koike T, et al. Neurotoxicity of advanced glycation end-products for cultured cortical neurons. J Neuropathol Exp Neurol (2000) 59:1094-105. doi:10.1093/ jnen/59.12.1094

24. Yamagishi S, Nakamura K, Inoue H, Kikuchi S, Takeuchi M. Serum or cerebrospinal fluid levels of glyceraldehyde-derived advanced glycation end products (AGEs) may be a promising biomarker for early detection of Alzheimer's disease. Med Hypotheses (2005) 64:1205-7. doi:10.1016/j.mehy.2005. 01.016

25. Prasad K. Low levels of serum soluble receptors for advanced glycation end products, biomarkers for disease state: myth or reality. Int J Angiol (2014) 23:11-6. doi:10.1055/s-0033-1363423

26. Flatow J, Buckley P, Miller BJ. Meta-analysis of oxidative stress in schizophrenia. Biol Psychiatry (2013) 74:400-9. doi:10.1016/j.biopsych.2013.03.018

27. Haack M, Hinze-Selch D, Fenzel T, Kraus T, Kuhn M, Schuld A, et al. Plasma levels of cytokines and soluble cytokine receptors in psychiatric patients upon hospital admission: effects of confounding factors and diagnosis. J Psychiatr Res (1999) 33:407-18. doi:10.1016/S0022-3956(99)00021-7

28. Muller N, Ackenheil M. Psychoneuroimmunology and the cytokine action in the CNS: implications for psychiatric disorders. Prog Neuropsychopharmacol Biol Psychiatry (1998) 22:1-33. doi:10.1016/S0278-5846(97)00179-6

29. Pae CU. Potential role of lymphotoxin-alpha (tumor necrosis factor-beta) in the development of schizophrenia. Med Hypotheses (2007) 68:1359-62. doi:10.1016/j.mehy.2006.10.023

30. Wassink TH, Crowe RR, Andreasen NC. Tumor necrosis factor receptor-II: heritability and effect on brain morphology in schizophrenia. Mol Psychiatry (2000) 5:678-82. doi:10.1038/sj.mp.4000807

31. Miller BJ, Buckley P, Seabolt W, Mellor A, Kirkpatrick B. Meta-analysis of cytokine alterations in schizophrenia: clinical status and antipsychotic effects. Biol Psychiatry (2011) 70:663-71. doi:10.1016/j.biopsych.2011.04.013

32. Idriss HT, Naismith JH. TNF alpha and the TNF receptor superfamily: structure-function relationship(s). Microsc Res Tech (2000) 50:184-95. doi:10.1002/1097-0029(20000801)50:3<184::AID-JEMT2>3.0.CO;2-H

33. Levine SJ, Adamik B, Hawari FI, Islam A, Yu ZX, Liao DW, et al. Proteasome inhibition induces TNFR1 shedding from human airway epithelial (NCIH292) cells. Am JPhysiol Lung Cell Mol Physiol (2005) 289:L233-43. doi:10.1152/ajplung.00469.2004

34. Coelho FM, Reis HJ, Nicolato R, Romano-Silva MA, Teixeira MM, Bauer ME, et al. Increased serum levels of inflammatory markers in chronic institutionalized patients with schizophrenia. Neuroimmunomodulation (2008) 15:140-4. doi:10.1159/000148197

35. Noto C, Gadelha A, Belangero SI, Spindola LM, Rocha NP, de Miranda AS, et al. Circulating levels of sTNFR1 as a marker of severe clinical course in schizophrenia. JPsychiatr Res (2013) 47:467-71. doi:10.1016/j.jpsychires. 2012.12.010

36. Hope S, Melle I, Aukrust P, Steen NE, Birkenaes AB, Lorentzen S, et al. Similar immune profile in bipolar disorder and schizophrenia: selective increase in soluble tumor necrosis factor receptor I and von Willebrand factor. Bipolar Disord (2009) 11:726-34. doi:10.1111/j.1399-5618.2009.00757.x

37. Whitehead JP, Richards AA, Hickman IJ, Macdonald GA, Prins JB. Adiponectin - a key adipokine in the metabolic syndrome. Diabetes Obes Metab (2006) 8:264-80. doi:10.1111/j.1463-1326.2005.00510.x

38. Adachi H, Yanai H, Hirowatari Y. The underlying mechanisms for olanzapine-induced hypertriglyceridemia. JClin Med Res (2012) 4:206-8. doi:10.4021/jocmr802w

39. Bai YM, Chen JY, Yang WS, Chi YC, Liou YJ, Lin CC, et al. Adiponectin as a potential biomarker for the metabolic syndrome in Chinese patients taking clozapine for schizophrenia. J Clin Psychiatry (2007) 68:1834-9. doi:10.4088/ JCP.v68n1202

40. Balotsev R, Haring L, Koido K, Leping V, Kriisa K, Zilmer M, et al. Antipsychotic treatment is associated with inflammatory and metabolic biomarkers alterations among first-episode psychosis patients: a 7-month follow-up study. Early Interv Psychiatry (2017). doi:10.1111/eip.12457

41. Bartoli F, Crocamo C, Clerici M, Carra G. Second-generation antipsychotics and adiponectin levels in schizophrenia: a comparative meta-analysis. Eur Neuropsychopharmacol (2015) 25:1767-74. doi:10.1016/j. euroneuro.2015.06.011

42. Bartoli F, Lax A, Crocamo C, Clerici M, Carra G. Plasma adiponectin levels in schizophrenia and role of second-generation antipsychotics: a 
meta-analysis. Psychoneuroendocrinology (2015) 56:179-89. doi:10.1016/j. psyneuen.2015.03.012

43. Famulla S, Lamers D, Hartwig S, Passlack W, Horrighs A, Cramer A, et al. Pigment epithelium-derived factor (PEDF) is one of the most abundant proteins secreted by human adipocytes and induces insulin resistance and inflammatory signaling in muscle and fat cells. Int JObes (Lond) (2011) 35:762-72. doi:10.1038/ijo.2010.212

44. Kirch DG. Infection and autoimmunity as etiologic factors in schizophrenia: a review and reappraisal. Schizophr Bull (1993) 19:355-70. doi:10.1093/ schbul/19.2.355

45. Meyer U. Developmental neuroinflammation and schizophrenia. Prog Neuropsychopharmacol Biol Psychiatry (2013) 42:20-34. doi:10.1016/j.pnpbp. 2011.11.003

46. Brown AS. Further evidence of infectious insults in the pathogenesis and pathophysiology of schizophrenia. Am J Psychiatry (2011) 168:764-6. doi:10.1176/ appi.ajp.2011.11050722

47. Prasad KM, Watson AM, Dickerson FB, Yolken RH, Nimgaonkar VL. Exposure to herpes simplex virus type 1 and cognitive impairments in individuals with schizophrenia. Schizophr Bull (2012) 38:1137-48. doi:10.1093/schbul/sbs046

48. Prasad KM, Eack SM, Goradia D, Pancholi KM, Keshavan MS, Yolken RH, et al. Progressive gray matter loss and changes in cognitive functioning associated with exposure to herpes simplex virus 1 in schizophrenia: a longitudinal study. Am J Psychiatry (2011) 168:822-30. doi:10.1176/appi.ajp.2011.10101423

49. Howard JS III. Herpes encephalitis, schizophrenia and the crossroads of psychiatry. Integr Physiol Behav Sci (1996) 31:219-23. doi:10.1007/BF02691452

50. Takeuchi M, Yamagishi S. Possible involvement of advanced glycation end-products (AGEs) in the pathogenesis of Alzheimer's disease. Curr Pharm Des (2008) 14:973-8. doi:10.2174/138161208784139693

51. Yamagishi S, Matsui T, Nakamura K. Blockade of the advanced glycation end products (AGEs) and their receptor (RAGE) system is a possible mechanism for sustained beneficial effects of multifactorial intervention on mortality in type 2 diabetes. Med Hypotheses (2008) 71:749-51. doi:10.1016/j.mehy.2008.05.039

52. Yamagishi S, Matsui T, Nakamura K, Inoue H, Takeuchi M, Ueda S, et al. Olmesartan blocks inflammatory reactions in endothelial cells evoked by advanced glycation end products by suppressing generation of reactive oxygen species. Ophthalmic Res (2008) 40:10-5. doi:10.1159/000111152

53. Hanson DR, Gottesman II. Theories of schizophrenia: a genetic-inflammatory-vascular synthesis. BMC Med Genet (2005) 6:7. doi:10.1186/1471-2350-6-7
54. Muller N, Myint AM, Schwarz MJ. Inflammatory biomarkers and depression. Neurotox Res (2011) 19:308-18. doi:10.1007/s12640-010-9210-2

55. Goldsmith DR, Rapaport MH, Miller BJ. A meta-analysis of blood cytokine network alterations in psychiatric patients: comparisons between schizophrenia, bipolar disorder and depression. Mol Psychiatry (2016) 21:1696-709. doi:10.1038/mp.2016.3

56. Pedersen MS, Benros ME, Agerbo E, Borglum AD, Mortensen PB. Schizophrenia in patients with atopic disorders with particular emphasis on asthma: a Danish population-based study. Schizophr Res (2012) 138:58-62. doi:10.1016/j.schres.2012.02.019

57. Borovcanin M, Jovanovic I, Radosavljevic G, Djukic Dejanovic S, Stefanovic V, Arsenijevic N, et al. Antipsychotics can modulate the cytokine profile in schizophrenia: attenuation of the type-2 inflammatory response. Schizophr Res (2013) 147:103-9. doi:10.1016/j.schres.2013.03.027

58. Li B, Duan XH, Wu JF, Liu BJ, Luo QL, Jin HL, et al. Impact of psychosocial stress on airway inflammation and its mechanism in a murine model of allergic asthma. Chin Med J (2013) 126:325-34.

59. Hagen JM, Sutterland AL, Koeter MW, Lutter R, Cohen D, de Haan L. Advanced glycation end products in recent-onset psychosis indicate early onset of cardiovascular risk. J Clin Psychiatry (2017) 78:1395-401. doi:10.4088/ JCP.16m10972

60. Itokawa M, Miyashita M, Arai M, Dan T, Takahashi K, Tokunaga T, et al. Pyridoxamine: a novel treatment for schizophrenia with enhanced carbonyl stress. Psychiatry Clin Neurosci (2018) 72:35-44. doi:10.1111/pcn.12613

61. Bechter K. [The mild encephalitis-hypothesis - new findings and studies]. Psychiatr Prax (2004) 31(Suppl 1):S41-3. doi:10.1055/s-2004-828428

Conflict of Interest Statement: None of the authors have any conflicts of interest pertaining to this paper to disclose.

Copyright $\odot 2018$ Ohnuma, Nishimon, Takeda, Sannohe, Katsuta and Arai. This is an open-access article distributed under the terms of the Creative Commons Attribution License (CC BY). The use, distribution or reproduction in other forums is permitted, provided the original author(s) and the copyright owner are credited and that the original publication in this journal is cited, in accordance with accepted academic practice. No use, distribution or reproduction is permitted which does not comply with these terms. 